\title{
Evidence that the outcome of developmental expression of psychosis is worse for adolescents growing up in an urban environment
}

\author{
JANNEKE SPAUWEN ${ }^{1}$, LYDIA KRABBENDAM ${ }^{1}$, ROSELIND LIEB ${ }^{2}$, \\ HANS-ULRICH WITTCHEN ${ }^{2,3}$ AND JIM VAN OS ${ }^{1,4 *}$ \\ ${ }^{1}$ Department of Psychiatry and Neuropsychology, South Limburg Mental Health Research and Teaching \\ Network, EURON, Maastricht University, Maastricht, The Netherlands $;{ }^{2}$ Max Planck Institute of Psychiatry, \\ Clinical Psychology and Epidemiology Unit, Munich, Germany; ${ }^{3}$ Institute of Clinical Psychology and \\ Psychotherapy, Technical University Dresden, Dresden, Germany; ${ }^{4}$ Division of Psychological Medicine, \\ Institute of Psychiatry, De Crespigny Park, Denmark Hill, London, UK
}

\begin{abstract}
Background. The urban environment may increase the risk for psychotic disorder in interaction with pre-existing risk for psychosis, but direct confirmation has been lacking. The hypothesis was examined that the outcome of subclinical expression of psychosis during adolescence, as an indicator of psychosis-proneness, would be worse for those growing up in an urban environment, in terms of having a greater probability of psychosis persistence over a $3 \cdot 5$-year period.
\end{abstract}

Method. A cohort of 918 adolescents from the Early Developmental Stages of Psychopathology Study (EDSP), aged 14-17 years (mean 15·1 years), growing up in contrasting urban and non-urban environments, completed a self-report measure of psychotic symptoms at baseline (Baseline Psychosis) and at first follow-up around 1 year post-baseline (T1). They were again interviewed by trained psychologists for the presence of psychotic symptoms at the second follow-up on average $3 \cdot 5$ years post-baseline (T2).

Results. The rate of T2 psychotic symptoms was $14 \cdot 2 \%$ in those exposed to neither Baseline Psychosis nor Urbanicity, $12 \cdot 1 \%$ in those exposed to Urbanicity alone, $14.9 \%$ in those exposed to Baseline Psychosis alone and $29.0 \%$ in those exposed to both Baseline Psychosis and Urbanicity. The odds ratio (OR) for the combined exposure was $2 \cdot 46$ [95\% confidence interval (CI) 1·46-4·14], significantly greater than that expected if Urbanicity and Baseline Psychosis acted independently.

Conclusion. These findings support the suggestion that the outcome of the developmental expression of psychosis is worse in urban environments. The environment may impact on risk for psychotic disorder by causing an abnormal persistence of a developmentally common expression of psychotic experiences.

\section{INTRODUCTION}

There may be no other issue in schizophrenia research with more far-reaching public health implications than the finding that young people growing up in an urban environment

\footnotetext{
* Address for correspondence: Professor Jim Van Os, Department of Psychiatry and Neuropsychology, Maastricht University, PO Box 616 (DRT 10), 6200 MD Maastricht, The Netherlands.

(Email: j.vanos@sp.unimaas.nl)
}

accumulate an increased risk for schizophrenia (Lewis et al. 1992; Torrey et al. 1997; Marcelis et al. 1999 a; Mortensen et al. 1999; Van Os et al. 2001; Spauwen et al. 2004). It is likely that the kind of geographical variation in incidence associated with urbanization reflects an environmental exposure, and that this environment has its impact, through continuous or repeated exposure, on developing children and adolescents. Thus, the time window of exposure 
is not around the time of birth and not around the time of onset of psychotic disorder, but in between (Marcelis et al. 1999b; Pedersen \& Mortensen, 2001 a). Recent studies in Germany, Greece, the UK and The Netherlands (Van Os et al. 2001; Spauwen et al. 2004; Stefanis et al. $2004 a$ ) have shown that the increased level of risk for psychotic disorder in urban populations can also be observed at the level of isolated psychotic symptoms, independent of service use, sociodemographic factors including ethnic group, drug use and size of social network (Pedersen \& Mortensen, $2001 a, b$; Van Os et al. 2001). Recent analyses also suggest that the increased risk for psychotic disorder and psychotic symptoms in urban areas is not mediated by neuropsychological impairment, obstetric complications or childhood socio-economic position (Harrison et al. 2003; Stefanis et al. 2004a).

The urban exposure is unlikely to represent a non-causal epiphenomenon resulting from genetic selection, because changes in urban exposure states during childhood also result in changes in risk for the outcome of adult psychosis (Pedersen \& Mortensen, 2001a). There is evidence, however, that rather than selection, pre-existing vulnerability may play a role by making individuals more susceptible to the riskincreasing effect of the environmental factor associated with urbanicity (Van Os, 2004). If pre-existing vulnerability makes individuals more sensitive to the risk-increasing effect of the urban factor, those with the highest indices of vulnerability would be expected to be particularly susceptible. In two previous studies examining vulnerability-urbanicity interaction, family history of psychosis was used as an indicator of genetic liability, rather than a more direct indicator at the level of the person (Van Os et al. 2003, 2004). In the current study, we wanted to examine the association between a personal indicator of psychosis-proneness and the urban environment, in a sample of adolescents growing up in differentially urbanized environments. This design allows for a direct examination of the outcome of psychosisproneness in urban versus more rural environments.

The psychosis phenotype is thought to be expressed at levels well below its clinical manifestation, commonly referred to as psychosis-proneness, psychotic experiences, schizotypy or at-risk mental states (Meehl, 1962; Chapman et al. 1994; Kwapil et al. 1997; Verdoux et al. 1998 a; Vollema, 1999; Van Os et al. 2000; Stefanis et al. 2004b). Psychotic experiences are a suitable indicator of risk as they are associated with genetic risk for psychotic disorder (Miller et al. 2002) and have a high predictive value for later psychotic outcomes (Chapman et al. 1994; Poulton et al. 2000; Yung et al. 2003; Hanssen et al. 2005). In addition, recent work has shown that psychotic experiences, whether measured by self-report or by structured interview, show family-specific transmission in the general population (Hanssen et al. in press), and a twin study has reported that such familial clustering was due to shared genes (Linney et al. 2003). If subtle psychotic experiences can be considered as an indicator of risk and if there is an association between pre-existing risk and the urban environment, the outcome of psychotic experiences would be expected to be worse for young people growing up in an urban environment. We therefore tested the hypothesis that the outcome of psychotic experiences, in terms of their persistence, would be greater in adolescents growing up in an urban environment.

\section{SUBJECTS AND METHOD}

\section{Sample}

The Early Developmental Stages of Psychopathology (EDSP) Study (Lieb et al. 2000a) collected data on the prevalence, incidence, risk factors, co-morbidity and course of mental disorders in a random, representative population sample of adolescents and young adults (age range 14-24 years at baseline) in the Munich area (Germany). The overall design of the study is prospective (average 42 months), consisting of a baseline survey $(n=3021)$, two follow-up surveys and a family supplement. Fourteen- to 15 -year-olds were sampled at twice the rate of those aged 16-21, and 22- to 24-year-olds were sampled at half this rate. Because the primary aim of the study was to examine the incidence of psychopathology, the young group with the presumed highest incidence was included at twice the rate and the oldest group at half the rate. A complete and detailed description of design, sample, instruments, procedures and 
statistical methods of the EDSP is given elsewhere (Wittchen et al. 1998).

The baseline sample was drawn in 1994 from the government registries in Munich, Germany, of registrants expected to be 14-24 years of age at the time of the baseline interview in 1995 . Details about the sampling and representativeness of the whole EDSP sample, along with its sociodemographic characteristics, have been presented previously (Wittchen et al. 1998; Lieb et al. $2000 a$ ). A total of 3021 interviews were completed at baseline (T0; response rate $71 \%$ ). The first follow-up study (T1) was conducted only for respondents aged 14 to 17 years at T0, whereas the second follow-up study was conducted for all respondents. From the 3021 respondents of the T0 study, a total of 2548 interviews were completed at the second follow-up (T2), which occurred at an average of 42 months after T0 (response rate $84 \%$ ).

In the EDSP family supplement at T1, direct diagnostic interviews were conducted with the parents of the younger cohort (the 14- to 17-year-olds at T0) to obtain information about familial psychopathology (Lieb et al. 2000b). Primarily the mothers were interviewed. Fathers were interviewed only if the mother was not available (deceased or not located). The sample of parents thus consisted of 1053 individuals (1026 mothers and 27 fathers). Non-response in parents was predominantly because of refusal to participate $(12.9 \%)$, failure to contact parents $(0.7 \%)$, and lack of time $(0.5 \%)$.

For the current report, the risk set consisted of all subjects who were 14-17 years of age (the younger cohort) at $\mathrm{T} 0$ and had completed both the first and second follow-up and whose demographic and family history data were documented. This risk set $(n=918)$ was used as it allowed examination of individuals who were as much as possible within the previously demonstrated (Pedersen \& Mortensen, $2001 a$ ) window of exposure of the urban environment.

\section{Instruments}

Subjects were assessed with the computerassisted version of the Munich-Composite International Diagnostic Interview (M-CIDI) (Wittchen \& Pfister, 1997), an updated version of the World Health Organization's Composite International Diagnostic Interview version 1.2
(WHO, 1990). Diagnostic findings, according to the explicit diagnostic criteria of the DSM-IV (APA, 1994), were obtained by using the M-CIDI diagnostic algorithms. The CIDI is designed for use by trained interviewers who are not clinicians and has high inter-rater reliability (Cottler et al. 1991; Wittchen et al. 1991) and high test-retest reliability (Wittchen, 1994). The assessment of psychosis with CIDI interviews by lay interviewers is not considered reliable (Anthony et al. 1985). Therefore, in the EDSP, trained psychologists who were allowed to probe with follow-up clinical questions conducted the interviews. Most interviews were carried out in the homes of the respondents. At T0, the lifetime version of the M-CIDI was used. At each of the follow-up assessments, the M-CIDI interval version was applied, which refers to the period of assessment from the last interview until the present.

Data on the M-CIDI-G-section about psychosis were only collected at T2 assessment, when lifetime ratings of psychosis were made, yielding cumulative incidence data up to the respective age of respondents at $\mathrm{T} 2$. At $\mathrm{T} 0$, and again at T2, participants additionally completed the self-report Symptom Checklist-90-R (SCL-90-R) to screen for a broad range of psychological problems and symptoms of psychopathology. The SCL-90-R measures nine primary symptom dimensions and is designed to provide an overview of a patient's symptoms and their severity (symptoms are scored dimensionally on a severity scale from 0 to 4 ) in the past 2 weeks. Reliability and validity of the SCL-90-R have been established previously (Derogatis \& Cleary, 1977; Bonicatto et al. 1997).

\section{Baseline and T1 assessment of Baseline Psychosis}

The SCL-90-R subscales 'Psychoticism' and 'Paranoia' were used to measure liability for psychosis at baseline and at first follow-up (T0 and T1). These scales include self-reports on thought interference, hallucinations and suspiciousness, and can be regarded, if not as clearcut psychotic symptoms, as an expression of psychotic experiences or psychosis-proneness (items 7, 8, 16, 18, 35, 43, 62, 68, 76, 77, 83-85, 87, 88, 90). The 'Psychoticism' and 'Paranoia' scales were combined into one psychosis scale 
by adding their scores and dividing the sum by two, and the T0 and T1 psychosis scales were similarly combined into one $\mathrm{T} 0 / \mathrm{T} 1$ baseline psychosis measure. For the purposes of the analyses, 'SCL Baseline Psychosis' was $a$ priori defined dichotomously as the group of individuals with the highest $25 \%$ of scores.

\section{T2 psychosis outcome}

In the adolescents and young adults, the ratings from the 15 M-CIDI core psychosis items on delusions (11 items) and hallucinations (four items) were used to assess the presence of psychotic symptoms (items G3-G5, G7-G14, G17, G18, G20, G21). These concern classic psychotic experiences involving, for example, persecution, thought interference and auditory hallucinations. Subjects were first asked to read a list with all the psychotic experiences and then interviewed by the psychologist (list and phrasing available on request). Onset of psychotic symptoms was recorded as within the past year or before that time.

All psychosis items could be rated in two ways: 0 (no) and 1 (yes). The study was not powered for the study of rare psychotic disorders, but instead focused on the presence of positive psychotic experiences. The psychosis outcome in this study was defined as at least one positive rating on any of the $15 \mathrm{M}$-CIDI core psychosis items. This outcome will be referred to hereafter as the T2 CIDI psychosis outcome. More details on the methods have been described elsewhere (Lieb et al. 2000 a).

Urbanicity was defined as the German city of Munich versus the surroundings of Munich. The population density of the surroundings was 553 persons per square mile and that of the city was 4061 persons per square mile.

\section{Statistical analysis}

All standard errors and test statistics were estimated using the software package STATA, version 8 (StataCorp, 2003). Main effects were expressed as odds ratios (ORs) and their $95 \%$ confidence intervals (CIs) from the logistic regression procedure. Conforming to previous work (Van Os et al. 2002, 2003; Van Os, 2004), interaction was calculated under an additive model rather than a multiplicative model as only additive interaction can be interpreted biologically in a meaningful way, yielding information on the extent to which two causes depend on each other or co-participate in disease causation (Darroch, 1997). In the logistic regression model, Baseline Psychosis and Urbanicity are additively associated with the logit of the probability of onset but multiplicatively linked to the probability itself. We estimated additive interaction by comparing the ORs for the combined categories of SCL Baseline Psychosis and Urbanicity with what would be expected in the case of no interaction. If $R_{i, j}$ is the relative risk for exposures, $i$ and $j=1$ (present) or 0 (absent), then the additive model in the case of absence of interaction or independence of effects, with R0, 0 as the common reference category, is: $\mathrm{R} 1,1=\mathrm{R} 1,0+\mathrm{R} 0,1-1$ (Rothman, 1986). Assuming that the ORs are approximately equal to the relative risks (which is true for disorders with a reasonably low prevalence), absence of interaction would be OR $(1,1)=\mathrm{OR}$ $(1,0)+\mathrm{OR}(0,1)-1$. This latter equation was assessed by the Wald test [testing the null hypothesis of $\mathrm{OR}(1,1)-\mathrm{OR}(1,0)-\mathrm{OR}(0,1)+1=0]$, adjusting for gender, SES (socio-economic status: a combination of social status and financial status), any drug use (mainly cannabis) and a family history of psychotic disorder as reported by the parents, guided by previous literature in the choice of these covariates (Van Os et al. 2001, 2002).

As the prevalence of psychotic symptoms was high enough to possibly violate the assumption that the ORs are approximately equal to the relative risks, results were also calculated with an additive risk model rather than the additive relative risk model described above (Greenland, 1993). To this end, the BINREG procedure in the STATA statistical program, which fits generalized linear models for the binomial family estimating risk differences (risk difference regression), was used. Absence of interaction here, with risk difference $(0,0)$ as the common reference category, would correspond to: risk difference $(1,1)=$ risk difference $(1,0)+$ risk difference $(0,1)$.

As some adolescents may have reported T2 CIDI psychotic symptoms that they might have already had at the time of T0 and or T1, a sensitivity analysis was conducted excluding adolescents who had reported onset of T2 CIDI psychotic symptoms longer than 1 year ago, ensuring prediction of only incident psychotic symptoms. 
Table 1. Rates of T2 CIDI psychotic symptoms according to the four exposure states formed by Urbanicity (urban versus rural) and SCL Baseline Psychosis (high versus low)

\begin{tabular}{|c|c|c|}
\hline \multirow[b]{2}{*}{ Exposure status } & \multicolumn{2}{|c|}{ Urbanicity } \\
\hline & Rural & Urban \\
\hline \multicolumn{3}{|c|}{ SCL Baseline Psychosis } \\
\hline Low & $\begin{array}{l}14 \cdot 2 \%(30 / 211) \\
(\mathrm{OR} 1)\end{array}$ & $\begin{array}{l}12 \cdot 1 \%(58 / 481) \\
\text { (OR } 0 \cdot 83,95 \% \text { CI } 0 \cdot 52-1 \cdot 33)\end{array}$ \\
\hline High & $\begin{array}{l}14 \cdot 9 \%(11 / 74) \\
(\text { OR } 1 \cdot 05,95 \% \text { CI } 0 \cdot 50-2 \cdot 23)\end{array}$ & $\begin{array}{l}29 \cdot 0 \%(44 / 152) \\
(\text { OR } 2 \cdot 46,95 \% \text { CI } 1 \cdot 46-4 \cdot 14)\end{array}$ \\
\hline Test OR (Urban & hosis high) - OR (SCL Baseline & igh $)-$ OR $($ Urban $)+1=0: \chi^{2}=$ \\
\hline
\end{tabular}

OR, odds ratio; CI, confidence interval.

a Adjusted for gender, socio-economic status, drug use and a family history of psychotic disorder as reported by the parents.

\section{RESULTS}

As described earlier, the analysis was based on a total of 918 adolescents (472 males, $51 \%$ ) with a mean age of $15 \cdot 1$ years (s.D. $=1 \cdot 1)$ who completed the final follow-up investigation. The mean T0/T1 SCL Baseline Psychosis score was $0 \cdot 31$ (S.D. $=0 \cdot 30$ ). Of the 918 adolescents, 633 $(69 \%)$ were living in an urban environment. The presence of SCL Baseline Psychosis $(25 \%$ highest SCL Baseline Psychosis scores) was similar in rural and urban-dwelling adolescents (rural 26\%, urban 24\%, $\chi^{2}=0 \cdot 40, p=0.53$ ). One-hundred and forty-three adolescents (16\%) had ever had at least one T2 CIDI psychotic symptom. Of these $143,76 \%$ reported onset of symptoms within the past year.

\section{Urbanicity, SCL Baseline Psychosis and T2 CIDI psychotic symptoms}

The rates of $\mathrm{T} 2$ CIDI psychotic symptoms in the four exposure states are depicted in Table 1, together with their odds ratios. The rate of T2 psychotic symptoms was $14.2 \%$ in those exposed to neither SCL Baseline Psychosis nor Urbanicity, $12.1 \%$ in those exposed to Urbanicity alone, $14.9 \%$ in those exposed to SCL Baseline Psychosis alone and $29.0 \%$ in those exposed to both SCL Baseline Psychosis and Urbanicity. The OR for the combined exposure category was $2 \cdot 46$ (95\% CI $1 \cdot 46-4 \cdot 14)$, whereas for SCL Baseline Psychosis alone it was $1.05(95 \%$ CI $0 \cdot 50-2 \cdot 23)$ and for Urbanicity alone it was $0.83(95 \%$ CI $0.52-1.33)$. This suggests a departure from independence, as the expected OR in the case of independence would have been $0.88(1.05+0.83-1=0.88)$.
The formal test of OR(urban + high liability) $-\mathrm{OR}$ (high liability) $-\mathrm{OR}($ urban $)+1=0$ indicated significant interaction $\left(\chi^{2}=5 \cdot 5, p=0 \cdot 020\right.$; adjusted $\left.\chi^{2}=6 \cdot 2, p=0 \cdot 013\right)$. Excluding the $24 \%$ of adolescents with T2 CIDI psychotic symptoms with onset more than 1 year ago did not change the results $\left(\chi^{2}=4 \cdot 4, p=0 \cdot 037\right)$. Similarly, using the additive risk model rather than the additive relative risk model also yielded similar results $\left(\chi^{2}=6 \cdot 8, p=0 \cdot 009\right.$; adjusted $\chi^{2}=3 \cdot 5$, $p=0.06$; recent psychotic symptoms only $\left.\chi^{2}=12 \cdot 8, p=0 \cdot 0004\right)$.

\section{DISCUSSION}

The risk-increasing effect of urbanicity on the occurrence of psychotic symptoms was only apparent in those with pre-existing psychotic experiences, independent of other variables that are known to increase the risk for psychosis. This study, using psychometric measures of psychosis-proneness, provides a more direct confirmation of previous research (Van Os et al. 2003, 2004), where it was found that a proxy genetic risk factor for schizophrenia interacted synergistically with the proxy environmental risk factor that urbanicity represents, and that a substantial proportion of individuals exposed to both the environmental and the genetic risk factors developed the disorder because of their co-participation. To the extent that variation in measures of psychosis-proneness is due to genetic factors (Linney et al. 2003), the current findings may represent gene-environment interaction. However, to the extent that variation in measures of psychosis-proneness is due to environmental factors (Van Os et al. 2005), 
the findings may represent environmentenvironment interaction. The results are not due to person-environment correlation, as urbanicity was not associated with the SCL Baseline Psychosis measure.

The T0/T1 measure of SCL Baseline Psychosis was in fact an SCL-90-R self-report of psychotic experiences, whereas the T2 CIDI psychotic symptom outcome was based on the M-CIDI clinical interview. This leaves the findings open to two related but slightly different interpretations. First, transition from T0/T1 SCL Baseline Psychosis to T2 CIDI psychotic symptoms may reflect the transition from expression of psychosis-proneness at $\mathrm{T} 0 / \mathrm{T} 1$ to expression of more severe overt symptoms at $\mathrm{T} 2$. Second, if T0/T1 SCL-90-R psychotic experiences are considered to be conceptually identical to the T2 psychotic symptoms of the M-CIDI psychosis section, transition from T0/T1 SCL Baseline Psychosis to T2 CIDI psychotic symptoms reflects persistence of subclinical psychosis. In fact, either interpretation would be important and shed light on the mechanism whereby the urban environment increases the risk for psychosis. The conservative interpretation is that the outcome of the age-associated rise in expression of psychosis associated with puberty (Galdos et al. 1993), which is common and can be measured in the general population, is more likely to persist in an urban environment, whereas in rural environments the expression declines again with age and therefore has a better outcome. These findings may be important, as they suggest that the ontogenesis of psychosis may be conceived as the poor outcome of a developmentally common psychosis phenotype that, under the influence of environmental factors, may display abnormal persistence thus possibly increasing the risk for progression towards clinical psychotic disorder.

The fact that in these data SCL Baseline Psychosis had no main effect in the absence of the urban environment and vice versa that the urban environment had no main effect in the absence of SCL Baseline Psychosis suggests that these two exposures are heavily co-dependent on each other in influencing risk, and that previous reports of 'main' effects of these factors may in fact reflect unmeasured interaction. A pattern of complete interaction in the absence of main effects makes sense epidemiologically, as a rare disease such as schizophrenia can only be credibly linked to an extremely prevalent exposure such as urbanicity if strong interaction with a third factor is present.

A possible mechanism of risk may reside in behavioural and/or neurochemical sensitization. Living in an urban environment is associated with increased exposure to environmental stress (high traffic densities, neighbourhood noise, violence and victimization, reduced social capital and pollution). The enduring enhancement of the behavioural response to this environmental stress is described as behavioural sensitization (Myin-Germeys et al. $2005 a$ ). Behavioural sensitization in relation to environmental stress may be interpreted in the light of the dopamine (DA) sensitization hypothesis of psychotic symptoms. It has been suggested that schizophrenia is associated with endogenous DA sensitization, a state characterized by hyper-responsiveness of DA neurons to environmental stimuli in which even exposure to moderate levels of stress are associated with excessive DA (Laruelle, 2000; Kapur, 2003). Furthermore, it has been suggested that DA mediates the expression of psychotic experiences in patients with schizophrenia (Marcelis et al. 2004; Myin-Germeys et al. 2005b).

\section{Methodological issues}

Psychotic symptoms cannot be equated with psychotic disorder. Symptoms are more prevalent than DSM-IV-defined psychotic disorders (APA, 1994), but nevertheless show a degree of continuity with more severe states such as schizophrenia (Poulton et al. 2000; Johns \& Van Os, 2001; Yung et al. 2003). Apart from strong evidence of phenomenological continuity in form and structure (Vollema \& Van den Bosch, 1995; Gruzelier, 1996; Vollema \& Hoijtink, 2000; Mata et al. 2003), continuity between symptoms and disorder is further suggested by (i) similar associations with demographic factors in epidemiological studies, in particular the negative association with age and the positive association with single marital status and social disadvantage (Verdoux et al. 1998b; Peters et al. 1999; Van Os et al. 2000; Johns \& Van Os, 2001), (ii) transitions over time from symptoms to disorder, even over periods longer than 15 years (Chapman et al. 1994; Poulton et al. 2000; Hanssen et al. 2005), (iii) familial 
co-clustering of the clinical and the subclinical (Kendler et al. 1993), (iv) strong dose-response effects of the urban environment on both symptoms and disorder (Van Os et al. 2001; Stefanis et al. 2004a), (v) sharing of cognitive and motor deficits (Lenzenweger \& Korfine, 1994; Voglmaier et al. 2000; Neumann \& Walker, 2003; Matsui et al. 2004), although this may not hold for all cognitive measures (Lenzenweger \& Gold, 2000; Johnson et al. 2003), (vi) sharing of risk genes (Stefanis et al. $2004 c$ ) and sharing of environmental risk factors such as cannabis (Arseneault et al. 2002; Van Os et al. 2002; Fergusson et al. 2003).

Use of the SCL-90-R as a measure of baseline psychosis-proneness is a possible limitation, as assessment of the SCL covers only the past 2 weeks. This might have led to false negatives in the baseline assessment of liability to psychosis. However, any possible bias in the direction of false negatives would have only decreased risk differences between groups, suggesting even larger effects of baseline liability than observed in the present study.

Another limitation of this work concerns the use of the CIDI to assess psychosis at T2 (Anthony et al. 1985). However, the use of face-to-face interviewing by psychologists can be expected to yield a much better result than a self-report questionnaire. Furthermore, the psychologists were allowed to probe with follow-up clinical questions so that the respondents' answers cannot be taken to represent self-report, as would be the case in the event of lay interviewer assessments. In the M-CIDI, the assessment of the symptoms does not include the degree of distress or disability associated with the symptoms. Respondents who only have minor distress cannot be distinguished from respondents who have major distress. However, it has been demonstrated that psychotic or psychosis-like symptoms may occur in the population without any accompanying distress (Van Os et al. 2001).

A possible final limitation of the study is that urban or rural residence was used as the exposure variable and that it is not known whether place of residence was the same as place of growing up. However, given the fact that this was a sample of individuals who were either still growing up or who had just reached adulthood, the rate of exposure misclassification is likely to be low, and misclassification would have served to reduce rather than to increase the reported association. The fact that the difference between the urban and rural population densities was almost eightfold attests to the validity of the exposure, although it did not allow for examination of dose-response relationships.

\section{ACKNOWLEDGEMENTS}

This work is part of the EDSP Study and was funded by the German Ministry of Research and Technology, project nos 01EB9405/6, 01EB $9901 / 6$ and EB01016200. Part of the field work and analyses were also supported by grants from the Deutsche Forschungsgemeinschaft (DFG) LA1148/1-1 and WI2246/1-1. The principal investigators were Hans-Ulrich Wittchen and Roselind Lieb. Core staff members of the EDSP group are: Kirsten von Sydow, Gabriele Lachner, Axel Perkonigg, Peter Schuster, Michael Höfler, Holger Sonntag, Tanja Brückl, Elzbieta Garczynski, Barbara Isensee, Agnes Nocon, Chris Nelson, Hildegard Pfister, Victoria Reed, Barbara Spiegel, Andrea Schreier, Ursula Wunderlich and Petra Zimmermann. Scientific advisors are Jules Angst (Zurich), Jürgen Margraf (Basel), Günther Esser (Potsdam), Kathleen Merikangas (NIMH, Bethesda), Ron Kessler (Harvard, Boston) and Jim van Os (Maastricht).

\section{DECLARATION OF INTEREST}

None.

\section{REFERENCES}

Anthony, J. C., Folstein, M., Romanoski, A. J., Von Korff, M. R., Nestadt, G. R., Chahal, R., Merchant, A., Brown, C. H., Shapiro, S. \& Kramer, M. (1985). Comparison of the lay Diagnostic Interview Schedule and a standardized psychiatric diagnosis. Experience in eastern Baltimore. Archives of General Psychiatry 42, 667-675.

APA (1994). Diagnostic and Statistical Manual of Mental Disorders (4th edn). American Psychiatric Press: Washington, DC.

Arseneault, L., Cannon, M., Poulton, R., Murray, R., Caspi, A. \& Moffitt, T. E. (2002). Cannabis use in adolescence and risk for adult psychosis: longitudinal prospective study. British Medical Journal 325, 1212-1213.

Bonicatto, S., Dew, M. A., Soria, J. J. \& Seghezzo, M. E. (1997). Validity and reliability of Symptom Checklist '90 (SCL90) in an Argentine population sample. Social Psychiatry and Psychiatric Epidemiology 32, 332-338.

Chapman, L. J., Chapman, J. P., Kwapil, T. R., Eckblad, M. \& Zinser, M. C. (1994). Putatively psychosis-prone subjects 10 years later. Journal of Abnormal Psychology 103, 171-183. 
Cottler, L. B., Helzer, J. E., Mager, D., Spitznagel, E. L. \& Compton, W. M. (1991). Agreement between DSM-III and III-R substance use disorders. Drug and Alcohol Dependence 29, 17-25.

Darroch, J. (1997). Biologic synergism and parallelism. American Journal of Epidemiology 145, 661-668.

Derogatis, L. R. \& Cleary, P. A. (1977). Confirmation of the dimensional structure of the SCL-90: a study in construct validation. Journal of Clinical Psychology 33, 981-989.

Fergusson, D. M., Horwood, L. J. \& Swain-Campbell, N. R. (2003) Cannabis dependence and psychotic symptoms in young people. Psychological Medicine 33, 15-21.

Galdos, P. M., Van Os, J. J. \& Murray, R. M. (1993). Puberty and the onset of psychosis. Schizophrenia Research 10, 7-14.

Greenland, S. (1993). Additive risk versus additive relative risk models. Epidemiology 4, 32-36.

Gruzelier, J. H. (1996). The factorial structure of schizotypy: Part I. Affinities with syndromes of schizophrenia. Schizophrenia Bulletin 22, 611-620.

Hanssen, M., Bak, M., Bijl, R., Vollebergh, W. \& van Os, J. (2005). The incidence and outcome of subclinical psychotic experiences in the general population. British Journal of Clinical Psychology $\mathbf{4 4}$ 181-191.

Hanssen, M., Krabbendam, L., Vollema, M., Delespaul, P. \& Van Os, J. (in press). Evidence for instrument and family-specific variation of subclinical psychosis dimensions in the general population. Journal of Abnormal Psychology.

Harrison, G., Fouskakis, D., Rasmussen, F., Tynelius, P., Sipos, A. \& Gunnell, D. (2003). Association between psychotic disorder and urban place of birth is not mediated by obstetric complications or childhood socio-economic position: a cohort study. Psychological Medicine 33, 723-731.

Johns, L. C. \& Van Os, J. (2001). The continuity of psychotic experiences in the general population. Clinical Psychology Review 21, 1125-1141.

Johnson, J. K., Tuulio-Henriksson, A., Pirkola, T., Huttunen, M. O., Lonnqvist, J., Kaprio, J. \& Cannon, T. D. (2003). Do schizotypal symptoms mediate the relationship between genetic risk for schizophrenia and impaired neuropsychological performance in co-twins of schizophrenic patients? Biological Psychiatry 54, $1200-1204$

Kapur, S. (2003). Psychosis as a state of aberrant salience: a framework linking biology, phenomenology, and pharmacology in schizophrenia. American Journal of Psychiatry 160, 13-23.

Kendler, K. S., McGuire, M., Gruenberg, A. M., O'Hare, A., Spellman, M. \& Walsh, D. (1993). The Roscommon Family Study. III. Schizophrenia-related personality disorders in relatives. Archives of General Psychiatry 50, 781-788.

Kwapil, T. R., Miller, M. B., Zinser, M. C., Chapman, J. \& Chapman, L. J. (1997). Magical ideation and social anhedonia as predictors of psychosis proneness: a partial replication. Journal of Abnormal Psychology 106, 491-495.

Laruelle, M. (2000). The role of endogenous sensitization in the pathophysiology of schizophrenia: implications from recent brain imaging studies. Brain Research. Brain Research Reviews 31, 371-384.

Lenzenweger, M. F. \& Gold, J. M. (2000). Auditory working memory and verbal recall memory in schizotypy. Schizophrenia Research 42, 101-110.

Lenzenweger, M. F. \& Korfine, L. (1994). Perceptual aberrations, schizotypy, and the Wisconsin Card Sorting Test. Schizophrenia Bulletin 20, 345-357.

Lewis, G., David, A., Andreasson, S. \& Allebeck, P. (1992). Schizophrenia and city life. Lancet 340, 137-140.

Lieb, R., Isensee, B., von Sydow, K. \& Wittchen, H. U. (2000a). The Early Developmental Stages of Psychopathology Study (EDSP): a methodological update. European Addiction Research 6, 170-182.

Lieb, R., Wittchen, H. U., Hofler, M., Fuetsch, M., Stein, M. B. \& Merikangas, K. R. $(2000 b)$. Parental psychopathology, parenting styles, and the risk of social phobia in offspring: a prospectivelongitudinal community study. Archives of General Psychiatry 57, 859-866.
Linney, Y. M., Murray, R. M., Peters, E. R., MacDonald, A. M., Rijsdijk, F. \& Sham, P. C. (2003). A quantitative genetic analysis of schizotypal personality traits. Psychological Medicine 33, $803-816$

Marcelis, M., Cavalier, E., Gielen, J., Delespaul, P. \& Van Os, J. (2004). Abnormal response to metabolic stress in schizophrenia: marker of vulnerability or acquired sensitization? Psychological Medicine 34, 1103-1111.

Marcelis, M., Navarro-Mateu, F., Murray, R., Selten, J. P. \& Van Os, J. (1999a). Urbanization and psychosis: a study of 1942-1978 birth cohorts in The Netherlands. Psychological Medicine 28, 871-879.

Marcelis, M., Takei, N. \& van Os, J. (1999b). Urbanization and risk for schizophrenia: does the effect operate before or around the time of illness onset? Psychological Medicine 29, 1197-1203.

Mata, I., Gilvarry, C. M., Jones, P. B., Lewis, S. W., Murray, R. M. \& Sham, P. C. (2003). Schizotypal personality traits in nonpsychotic relatives are associated with positive symptoms in psychotic probands. Schizophrenia Bulletin 29, 273-283.

Matsui, M., Sumiyoshi, T., Kato, K., Yoneyama, E. \& Kurachi, M. (2004). Neuropsychological profile in patients with schizotypal personality disorder or schizophrenia. Psychological Report 94 387-397.

Meehl, P. E. (1962). Schizotaxia, schizotypy, schizophrenia. American Psychologist 17, 827-838.

Miller, P. M., Lawrie, S. M., Byrne, M., Cosway, R. \& Johnstone, E. C. (2002). Self-rated schizotypal cognitions, psychotic symptoms and the onset of schizophrenia in young people at high risk of schizophrenia. Acta Psychiatrica Scandinavica 105, 341-345.

Mortensen, P. B., Pedersen, C. B., Westergaard, T., Wohlfahrt, J., Ewald, H., Mors, O., Andersen, P. K. \& Melbye, M. (1999). Effects of family history and place and season of birth on the risk of schizophrenia. New England Journal of Medicine 340, 603-608.

Myin-Germeys, I., Delespaul, P. \& van Os, J. (2005a). Behavioural sensitization to daily life stress in psychosis. Psychological Medicine 35, 733-741.

Myin-Germeys, I., Marcelis, M., Krabbendam, L., Delespaul, P. \& van Os, J. (2005b). Subtle fluctuations in psychotic phenomena as functional states of abnormal dopamine reactivity in individuals at risk. Biological Psychiatry 58, 105-110.

Neumann, C. S. \& Walker, E. F. (2003). Neuromotor functioning in adolescents with schizotypal personality disorder: associations with symptoms and neurocognition. Schizophrenia Bulletin 29, 285-298.

Pedersen, C. B. \& Mortensen, P. B. (2001 a). Evidence of a doseresponse relationship between urbanicity during upbringing and schizophrenia risk. Archives of General Psychiatry 58, 1039-1046.

Pedersen, C. B. \& Mortensen, P. B. (2001 b). Family history, place and season of birth as risk factors for schizophrenia in Denmark: a replication and reanalysis. British Journal of Psychiatry 179, 4652.

Peters, E. R., Joseph, S. A. \& Garety, P. A. (1999). Measurement of delusional ideation in the normal population: introducing the PDI (Peters et al. Delusions Inventory). Schizophrenia Bulletin 25, 553-576.

Poulton, R., Caspi, A., Moffitt, T. E., Cannon, M., Murray, R. \& Harrington, H. (2000). Children's self-reported psychotic symptoms and adult schizophreniform disorder: a 15-year longitudinal study. Archives of General Psychiatry 57, 1053-1058.

Rothman, K. J. (1986). Interactions between causes, pp. 311-326. In Modern Epidemiology. Little, Brown and Company: Boston, MA.

Spauwen, J., Krabbendam, L., Lieb, R., Wittchen, H. U. \& van Os, J. (2004). Does urbanicity shift the population expression of psychosis? Journal of Psychiatric Research 38, 613-618.

StataCorp (2003). STATA statistical software: Release 8.0. Stata Corporation: College Station, TX, USA.

Stefanis, N., Delespaul, P., Smyrnis, N., Lembesi, A., Avramopoulos, D., Evdokimidis, I., Stefanis, C. \& van Os, J. (2004a). Is the excess 
risk of psychosis-like experiences in urban areas attributable to altered cognitive development? Social Psychiatry and Psychiatric Epidemiology 39, 364-368.

Stefanis, N. C., Smyrnis, N., Avramopoulos, D., Evdokimidis, I., Ntzoufras, I. \& Stefanis, C. N. (2004b). Factorial composition of self-rated schizotypal traits among young males undergoing military training. Schizophrenia Bulletin 30, 335-350.

Stefanis, N. C., Van Os, J., Avramopoulos, D., Smyrnis, N., Evdokimidis, I., Hantoumi, I. \& Stefanis, C. N. (2004c). Variation in catechol-o-methyltransferase val158 met genotype associated with schizotypy but not cognition: a population study in 543 young men. Biological Psychiatry 56, 510-515.

Torrey, E. F., Bowler, A. E. \& Clark, K. (1997). Urban birth and residence as risk factors for psychoses: an analysis of 1880 data. Schizophrenia Research 25, 169-176.

Van Os, J. (2004). Does the urban environment cause psychosis? British Journal of Psychiatry 184, 287-288.

Van Os, J., Bak, M., Hanssen, M., Bijl, R. V., de Graaf, R. \& Verdoux, H. (2002). Cannabis use and psychosis: a longitudinal population-based study. American Journal of Epidemiology $\mathbf{1 5 6}$, 319-327.

Van Os, J., Hanssen, M., Bak, M., Bijl, R. V. \& Vollebergh, W. (2003). Do urbanicity and familial liability coparticipate in causing psychosis? American Journal of Psychiatry 160, 477-482.

Van Os, J., Hanssen, M., Bijl, R.-V. \& Ravelli, A. (2000). Straus (1969) revisited: a psychosis continuum in the general population? Schizophrenia Research 45, 11-20.

Van Os, J., Hanssen, M., Bijl, R. V. \& Vollebergh, W. (2001). Prevalence of psychotic disorder and community level of psychotic symptoms: an urban-rural comparison. Archives of General Psychiatry 58, 663-668.

Van Os, J., Krabbendam, L., Myin-Germeys, I. \& Delespaul, P. (2005). The schizophrenia envirome. Current Opinion in Psychiatry 18, $141-145$.

Van Os, J., Pedersen, C. B. \& Mortensen, P. B. (2004). Confirmation of synergy between urbanicity and familial liability in the causation of psychosis. American Journal of Psychiatry 161, $2312-2314$.

Verdoux, H., Maurice-Tison, S., Gay, B., Van Os, J., Salamon, R. \& Bourgeois, M. L. (1998a). A survey of delusional ideation in primary-care patients. Psychological Medicine 28, 127-134.
Verdoux, H., Van Os, J., Maurice-Tison, S., Gay, B., Salamon, R. \& Bourgeois, M. (1998 b). Is early adulthood a critical developmental stage for psychosis proneness? A survey of delusional ideation in normal subjects. Schizophrenia Research 29, 247-254.

Voglmaier, M. M., Seidman, L. J., Niznikiewicz, M. A., Dickey, C. C., Shenton, M. E. \& McCarley, R. W. (2000). Verbal and nonverbal neuropsychological test performance in subjects with schizotypal personality disorder. American Journal of Psychiatry 157, 787-793.

Vollema, M. (1999). Schizotypy: Toward the Psychological Heart of Schizophrenia. Shaker: Groningen.

Vollema, M. G. \& Hoijtink, H. (2000). The multidimensionality of self-report schizotypy in a psychiatric population: an analysis using multidimensional Rasch models. Schizophrenia Bulletin $\mathbf{2 6}$ 565-575.

Vollema, M. G. \& Van den Bosch, R. J. (1995). The multidimensionality of schizotypy. Schizophrenia Bulletin 21, 19-31.

WHO (1990). Composite International Diagnostic Interview (CIDI), Version 1.0. World Health Organization: Geneva.

Wittchen, H. U. (1994). Reliability and validity studies of the WHO Composite International Diagnostic Interview (CIDI): a critical review. Journal of Psychiatric Research 28, 57-84.

Wittchen, H. U., Perkonigg, A., Lachner, G. \& Nelson, C. B. (1998). Early Developmental Stages of Psychopathology Study (EDSP): objectives and design. European Addiction Research 4, 18-27.

Wittchen, H.-U. \& Pfister, H. (1997). DIA-X-Interviews: Manual für Screening-Verfahren und Interview; Interviewheft Längsschnittuntersuchung (DIA-X-Lifetime); Ergänzungsheft (DIA-X-Lifetime); Interviewheft Querschnittsuntersuchung (DIA-X-12 MonatsVersion); Ergänzungsheft (DIA-X-12 Monats-Version); $P C$ Programm zur Durchführung der Interviews (Längsund Querschnittsuntersuchung). Auswertungsprogramm. Swets \& Zeitlinger: Frankfurt, Germany.

Wittchen, H. U., Robins, L. N., Cottler, L. B., Sartorius, N., Burke, J. D. \& Regier, D. (1991). Cross-cultural feasibility, reliability and sources of variance of the Composite International Diagnostic Interview (CIDI). The Multicentre WHO/ADAMHA Field Trials. British Journal of Psychiatry 159, 645-653.

Yung, A. R., Phillips, L. J., Yuen, H. P., Francey, S. M., McFarlane, C. A., Hallgren, M. \& McGorry, P. D. (2003). Psychosis prediction: 12-month follow-up of a high-risk ('prodromal') group. Schizophrenia Research 60, 21-32. 